\title{
Tuning the Magnetic Coupling of a Molecular Spin Interface via Electron Doping
}

\author{
Giulia Avvisati, Pierluigi Gargiani, Carlo Mariani, and Maria Grazia Betti* \\ Cite This: Nano Lett. 2021, 21, 666-672 \\ Read Online
}

ABSTRACT: Mastering the magnetic response of molecular spin interfaces by tuning the occupancy of the molecular orbitals, which carry the spin magnetic moment, can be accomplished by electron doping. We propose a viable route to control the magnetization direction and magnitude of a molecular spin network, in a graphene-mediated architecture, achieved via alkali doping of manganese phthalocyanine $(\mathrm{MnPc})$ molecules assembled on cobalt intercalated under a graphene membrane. The antiparallel magnetic alignment of the MnPc molecules with the underlying Co layer can be switched to a ferromagnetic state by electron doping. Multiplet calculations unveil an enhanced magnetic state of the Mn centers with a $3 / 2$

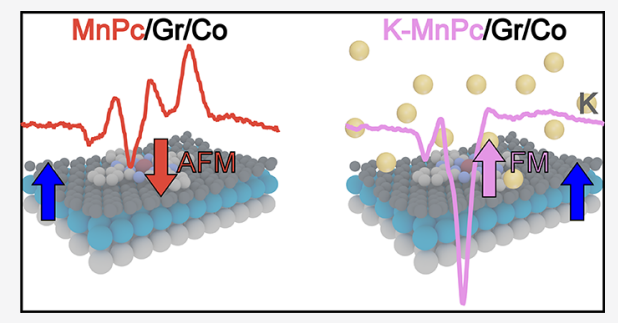
to $5 / 2$ spin transition induced by alkali doping, as confirmed by the steepening of the hysteresis loops, with higher saturation magnetization values. This new molecular spin configuration can be aligned by an external field, almost independently from the hardmagnet substrate effectively behaving as a free magnetic layer.

KEYWORDS: spin interface, graphene, magnetic molecule, alkali metal, X-ray magnetic circular dichroism

\section{INTRODUCTION}

Molecular-based magnets can exhibit superior properties compared with their inorganic counterparts, in terms of chemical stability and tuning. The possibility of molecular spin manipulation and tuning are attractive abilities, especially with respect to molecular spintronics. ${ }^{1,2}$ Stemming from the principle of using molecular semiconducting films as a spintransport media, several proof-of-concept devices have been realized, such as organic spin valves ${ }^{3-5}$ and organic magnetic tunnel junctions. ${ }^{6,7}$ In more recent experiments the role of the organic layer-magnetic electrode interface, the so-called spin interface, ${ }^{8}$ has become apparent, where it has been directly related to the magnetoresistance sign in organic spin valves ${ }^{9,10}$ and also to the insurgence of new phenomena such as interface spin harvesting. ${ }^{11,12}$ Thus, it turns out that a challenge and an opportunity to tailor the properties of organic-spintronic devices is to control the interface hybridization and magnetic coupling between organic molecules and magnetic electrodes. ${ }^{1,13}$ Recent endeavors to master the magnetic coupling between spin-carrying organic molecules and magnetic layers were achieved, tuning the magnetic response with external stimuli or chemical doping and changing the occupancy of the electronic molecular states carrying the spin. ${ }^{14-17}$ Spin manipulation of molecular interfaces with a designed architecture allows us to attain the goal of controlling the ferromagnetic (FM) or antiferromagnetic (AFM) coupling with a magnetic substrate, up to room temperature.

Transition metal phthalocyanines (TM-Pcs, TM $\mathrm{C}_{32} \mathrm{H}_{16} \mathrm{~N}_{8}$ ) are a class of molecules with an intriguing outlook in spintronics, thanks to the wide range of spin ground state configurations attainable by changing the central TM core ${ }^{18,19}$ or by chemical modification of the molecule, ${ }^{20}$ e.g., by electron doping via alkali metal ${ }^{21,22}$ or oxygen exposure. ${ }^{23-26}$ Electron doping of transition-metal phthalocyanines is a viable way to tune orbital occupation and symmetry, both in free-standinglike molecules ${ }^{27-31}$ and at the interface with a metallic support. $^{32}$ Furthermore, selectivity of the spin-polarized molecular orbital symmetry can allow us to tune the sign (and strength) of the magnetic coupling (FM or AFM) with a magnetic substrate. ${ }^{33}$

Recently, it has been demonstrated that an advanced organic spin-interface architecture constituted by MPc molecules magnetically coupled with metallic Co layers through a graphene (Gr) spacer presents magnetic remanence even at room temperature. ${ }^{33,34}$ Graphene acts as a protective membrane for the magnetic metal substrate and preserves the electronic/magnetic structure of the molecule, hindering hybridization/interaction of the spin-polarized molecular orbitals. ${ }^{19,34,35}$ This class of spin interfaces, stable against thermal fluctuations with preserved magnetic states thanks to the graphene spacer, can be a playground to tune and switch the AFM or FM coupling, not only by direct exchange between the molecule and the magnetic substrate but also by exploiting

Received: October 25, 2020

Revised: December 17, 2020

Published: December 23, 2020 


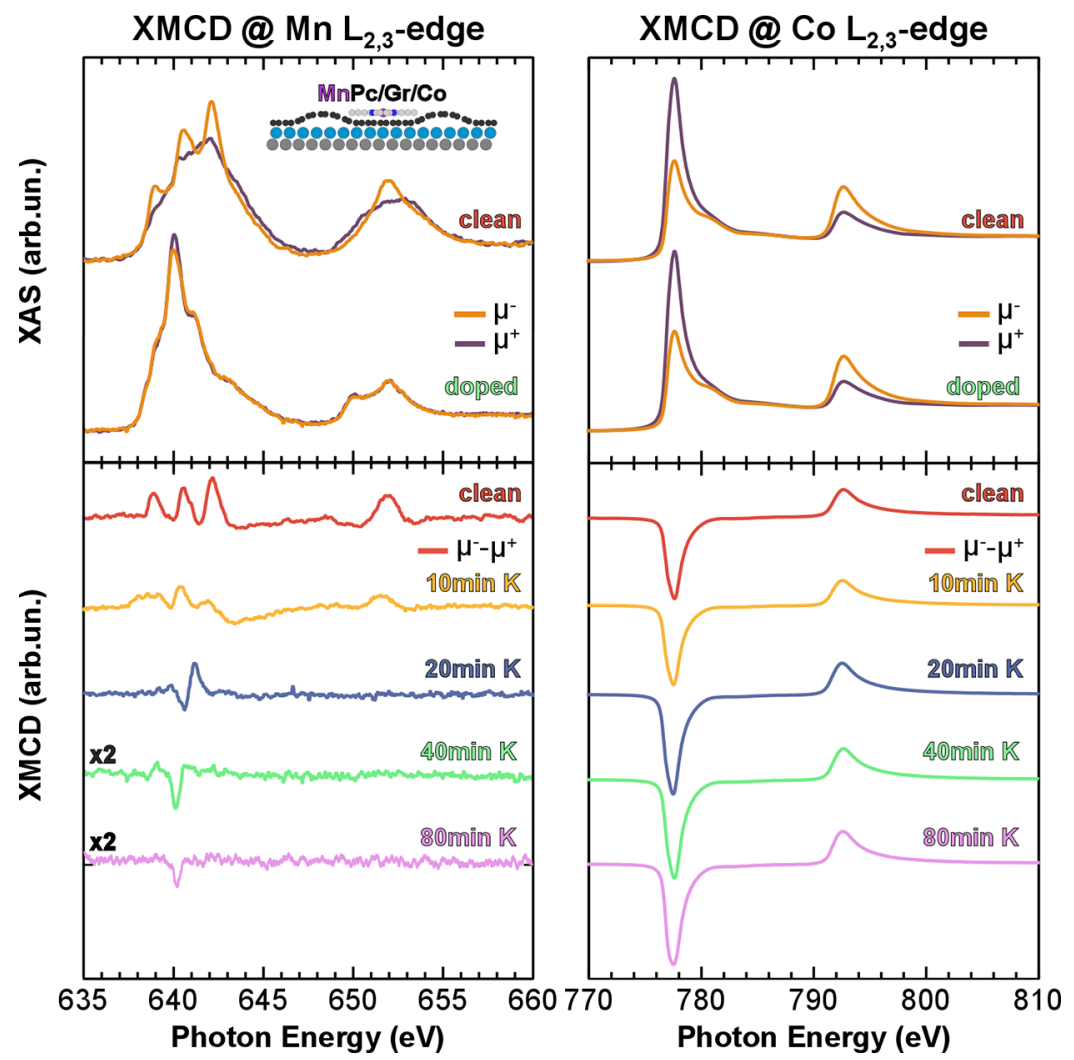

Figure 1. Top panels: XAS spectra acquired at the $\mathrm{Mn}$ (left) and Co (right) $\mathrm{L}_{2,3}$ edges with circularly polarized radiation for the $\mathrm{MnPc} / \mathrm{Gr} / 1 \mathrm{ML}$ $\mathrm{Co} / \mathrm{Ir}(111)$ sample before (clean) and after (doped) $40 \mathrm{~min}$ of $\mathrm{K}$ exposure. Bottom panels: XMCD spectra from low $\mathrm{K}$ coverage to saturation $\mathrm{K}$ coverage ( $80 \mathrm{~min} \mathrm{~K}$ deposition) are also reported. All the measurements were performed at a low sample temperature ( $3 \mathrm{~K})$, in remanence condition, and with polarized photons impinging the sample at normal incidence.

the indirect ligand-mediated super-exchange interaction. Tuning the coupling strength between the molecular film and the magnetic substrate is a fundamental step to enable the design of molecular spin interfaces responsive to small external magnetic stimuli, where a low magnetic coupling energy and high magnetic polarizability are both desirable properties. ${ }^{9,36}$

Herein, we report on the tuning of the magnetic response of $\mathrm{MnPc}$ molecules coupled with a single Co layer, intercalated under graphene, at increasing electron doping, via deposition of $\mathrm{K}$. The magnetic coupling, monitored via element-specific $\mathrm{X}$-ray magnetic circular dichroism (XMCD) measurements, switches from a strong AFM, with an exchange energy of $6.2 \pm$ $0.5 \mathrm{meV} /$ atom $^{33,34}$ to a field-polarizable FM state, as a result of a new molecular ground state revealed by multiplet calculations. The molecular spin interface upon electron doping presents a high magnetic polarizability mostly independent from the hard-magnet substrate, enabling possible applications in molecular ultra-thin spin-valve devices or multi-spin-interface designs with tailored magnetic response. $^{36}$

\section{RESULTS AND DISCUSSION}

Graphene-spaced spin molecular interfaces constituted by $\mathrm{MnPc}$ molecules adsorbed on a cobalt layer intercalated under graphene have been recently magnetically characterized. ${ }^{33-35}$ XAS absorption with left and right circularly polarized radiation in remanence conditions, together with their XMCD difference at $\mathrm{Mn}$ and $\mathrm{Co}_{2,3}$ metal edges for a single layer of $\mathrm{MnPc}$ molecules adsorbed on the graphene membrane on a Co intercalated layer, before and after exposure to potassium, are reported in Figure 1. The dichroic signal, reported in the lower panel of Figure 1, enlightens an antiparallel coupling of $\mathrm{Mn}$ ion cores and the Co layer, before doping. The occupancy and symmetry of the MnPc molecular orbitals carrying the magnetic moment automatically select the sign of the AFM alignment between the MnPc central ions and the Co layer. The graphene membrane and the organic cage of the MPcs play an active role and mediate the magnetic interaction. ${ }^{33}$ In fact, the AFM coupling of the MnPc molecules with the Co layer is driven by a superexchange $\mathrm{Co}-\mathrm{Gr}-\mathrm{N}-\mathrm{Mn} 180^{\circ}$ channel, efficient to turn on a robust AFM alignment of the molecules with the Co layer, stable even at room temperature. ${ }^{34}$

Electron doping at the $\mathrm{MnPc} / \mathrm{Co}$-intercalated $\mathrm{Gr}$ interface may alter the charge density distribution of the frontier molecular orbitals, hence reshaping the symmetry-matching criteria of the super-exchange interaction path and opening different magnetic coupling channels. This can be accomplished by $\mathrm{K}$ doping of $\mathrm{MnPc}$ molecules assembled on the rippled $\mathrm{Gr} / \mathrm{Co} / \mathrm{Ir}(111)$ interface. ${ }^{34,35}$ An accurate monitoring of the molecular magnetic state, at increasing $\mathrm{K}$ doping, has been performed to follow the effects of changing the molecular orbital occupancy and symmetry. The absorption spectra, measured at $\mathrm{Mn}$ and $\mathrm{Co}_{2,3}$ in remanence conditions, together with their XMCD difference at increasing $\mathrm{K}$ deposition time, are reported in Figure 1.

The XAS spectra at the $M n L_{2,3}$ edges show a complex line shape, due to the intermediate $S=3 / 2$ spin configuration with a rich multiplet structure. Upon electron doping the structured three-peaked XMCD Mn $\mathrm{L}_{3}$ signal of the $\mathrm{Mn}$ ions is replaced 
by a sharp feature centered at $640.0 \mathrm{eV}$. On the other hand, the $\mathrm{XMCD}$ signal at the Co $\mathrm{L}_{2,3}$ absorption edges is almost unaltered upon $\mathrm{K}$ doping. The $\mathrm{Mn}_{2,3} \mathrm{XMCD}$ in remanence state in the undoped state presents an opposite sign with respect to the Co one (AFM alignment); it decreases at the first $\mathrm{K}$ deposition stage, and then, it reverses and becomes parallel to the one of $\mathrm{Co}$ at increasing $\mathrm{K}$ doping. At saturation $\mathrm{K}$ coverage, the XMCD signals for $\mathrm{Mn}$ and $\mathrm{Co}$ are both negative, suggesting an FM magnetic coupling. Furthermore, it is worth noting that the intensity of the XMCD in the remanent state is strongly reduced in the $\mathrm{K}$-doped $\mathrm{MnPc}$, indicating a decrease of the magnetic coupling strength between the $\mathrm{Mn}$ ion and the Co layer.

The evolution is even more striking if a magnetic field $(B=6$ $\mathrm{T}$ ) is applied perpendicular to the surface plane (i.e., along the easy magnetization axis of $\left.\mathrm{MnPc} / \mathrm{Gr} / \mathrm{Co}^{34}\right)$. The XMCD spectra, acquired at the $\mathrm{Mn} \mathrm{L}_{3}$ absorption edge as a function of $\mathrm{K}$ coverage, with $B=6 \mathrm{~T}$ (solid lines) and in remanence conditions (dashed lines), are reported in the left panel of Figure 2. It is worth noting that the magnetic dichroism

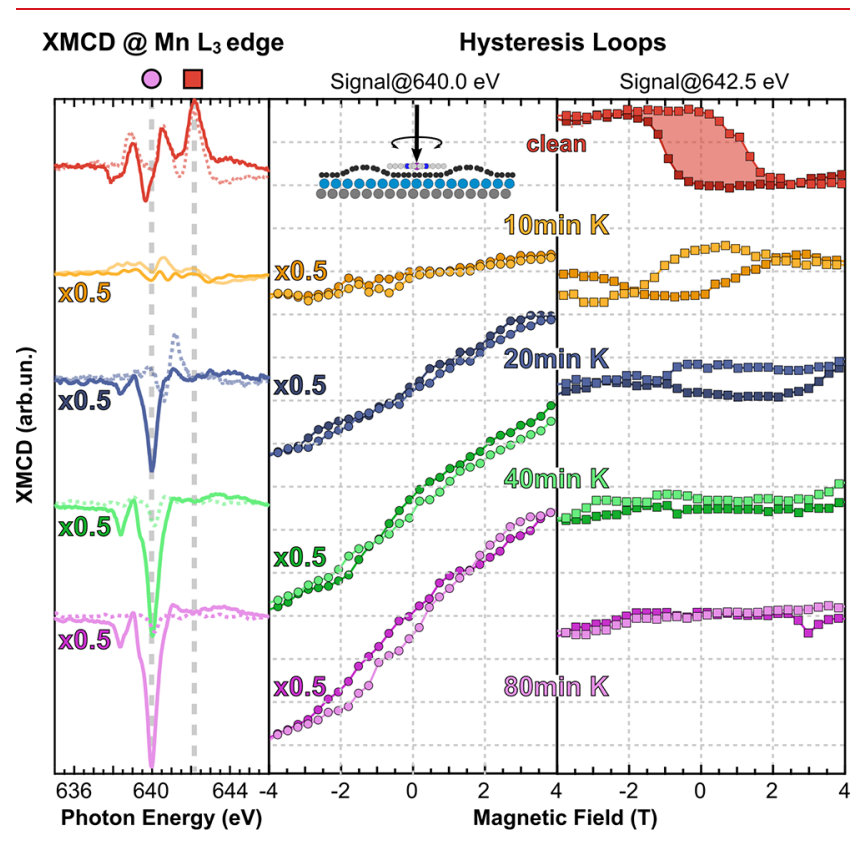

Figure 2. Left: $\mathrm{XMCD}$ measurements at the $\mathrm{Mn} \mathrm{L}_{2,3}$ edges acquired at normal incidence and low sample temperature $(3 \mathrm{~K})$, with (continuous lines) and without (dashed lines) the $6 \mathrm{~T}$ magnetic field. Center, right: hysteresis loops evolution at different doping levels. The magnetization curves were acquired by monitoring the field dependence of the XMCD signal at $642.5 \mathrm{eV}$ (i.e., maximum $\mathrm{XMCD}$ intensity for the clean system, right) and at $640.0 \mathrm{eV}$ (i.e., maximum XMCD intensity for the doped system, center).

measured at $B=6 \mathrm{~T}$, after a quenching at low $\mathrm{K}$ doses $(10 \mathrm{~min}$ $\mathrm{K})$, is strongly enhanced by increasing the $\mathrm{K}$ dosing up to saturation. To unveil the driving forces of this magnetic activity, the hysteresis loops have been measured by monitoring the field-dependent XMCD signal, both at the energy where the dichroic signal for the undoped system is maximum $(E=642.5 \mathrm{eV})$, and at the energy where the XMCD signal is maximum after $\mathrm{K}$ doping and almost zero for the pristine undoped one $(E=640.0 \mathrm{eV})$. The corresponding magnetization curves are reported in the right and central panels of Figure 2, respectively. Following the signal at 642.5 $\mathrm{eV}$, the magnetization curve reduces its amplitude at the early
$\mathrm{K}$ deposition stages, and it is quenched at saturation $\mathrm{K}$ coverage. On the other hand the hysteresis loops at $640 \mathrm{eV}$ become more intense as the $\mathrm{K}$ dosing is increased, indicating a higher susceptibility of the $\mathrm{Mn}$ ion. The magnetic response of the $\mathrm{MnPc}$ molecule to the external field is thus abruptly changed; in the pristine layer the Mn-ion magnetic moment is locked anti-parallel to the one of the Co film by the strong AF super-exchange coupling, up to an applied field of $6 \mathrm{~T},{ }^{34}$ and in the doped phase the molecular spin can be oriented by an external field, showing a weak FM coupling to the Co film in remanence condition.

This K-dosing-induced magnetic-state change may be attributed to electronic charge donated from $\mathrm{K}$ adatoms to the out-of-plane orbitals located on Mn ions. A direct electron doping of the $\mathrm{Mn}$ orbitals would alter the $\mathrm{Mn}$ orbital occupation and accordingly the metal-ion spin-orbital configuration. In order to address the evolution of the electronic structure of the central $\mathrm{Mn}$ ions upon $\mathrm{K}$ doping, $\mathrm{Mn} \mathrm{L}_{2,3}$ absorption edges acquired with linearly polarized light, with the polarization vector either parallel (in-plane, left) or nearly perpendicular (out-of-plane, right) to the surface plane are displayed in Figure 3 (left panels). The pristine MnPc single layer exhibits the typical electronic multiplet features of a MnPc thick molecular film, ${ }^{34,37,38}$ confirming an effective decoupling from the Co layer thanks to the graphene spacer.

The spectral line shape is markedly altered upon $\mathrm{K}$ doping, particularly for the in-plane $\mathrm{Mn}$ ion states. Indeed, the characteristic multiplet structure of the $\mathrm{Mn} \mathrm{L}_{3}$ edge in the in-plane polarized spectrum narrows already at the first $\mathrm{K}$ exposure, i.e., before the switch of the sign of the magnetic dichroic remanence. The out-of-plane spectrum of the undoped $\mathrm{MnPc} / \mathrm{Gr} / \mathrm{Co} / \operatorname{Ir}(111)$ is less structured and undergoes a similar evolution upon $\mathrm{K}$ deposition, presenting a similar single-featured line shape centered at $640 \mathrm{eV}$ at $\mathrm{K}$ saturation coverage. The total intensity of the empty Mn 3d states evaluated as the integral of the $\mathrm{L}_{3}+\mathrm{L}_{2}$ signal exhibits only a tiny reduction upon $\mathrm{K}$ doping (less than $10 \%$ of the normalized $\mathrm{L}_{2,3}$ integral), indicating that the $3 \mathrm{~d}$ ion electron occupancy is slightly affected by $\mathrm{K}$ adsorption, without a consistent charge transfer. Accordingly, a direct $\mathrm{e}^{-}$transfer to $\mathrm{Mn}$ molecular orbitals as a consequence of $\mathrm{K}$ deposition can be excluded. It is worth noting that the $\mathrm{L}_{3}$ peak spectral weight has substantially higher total intensity for the K-doped $\mathrm{MnPc}$, while the $\mathrm{L}_{2}$ is reduced, suggesting a transition to a higher spin state as deduced by the increased $\mathrm{L}_{3} /\left(\mathrm{L}_{3}+\mathrm{L}_{2}\right)$ branching ratio. ${ }^{39}$

Moving from the central $\mathrm{Mn}$ ion to the ligand states of the surrounding organic cage to ascertain the recipient of the electron donation, we report in Figure 3 (right panel) the $\mathrm{N} \mathrm{K}$ edge XAS, as a function of $\mathrm{K}$ dosing. The $\mathrm{N}$ K-edge $\pi^{*}$ transitions of the pristine molecule present four features, where the first two are associated with core level transitions form the two unequivalent nitrogen atoms of the molecule ${ }^{40}$ while the other two at 401 and $403 \mathrm{eV}$ are associated with macrocycle molecular states. Upon initial $\mathrm{K}$ dosing, the lower energy $\pi^{*}$ feature undergoes a substantial intensity reduction, suggesting a charge donation to $\mathrm{N}-\mathrm{Mn}$ hybridized orbitals. Further $\mathrm{K}$ deposition induces an energy shift toward lower photon energies of the macrocycle-related peaks, indicating a charge donation process involving the organic ligand cage. The modification of the linear dichroism can be associated with a partially broken symmetry of the $\pi^{*}$ and $\sigma^{*}$ resonances, induced by the adsorption of $\mathrm{K}$ adatoms. In fact, a distorted 


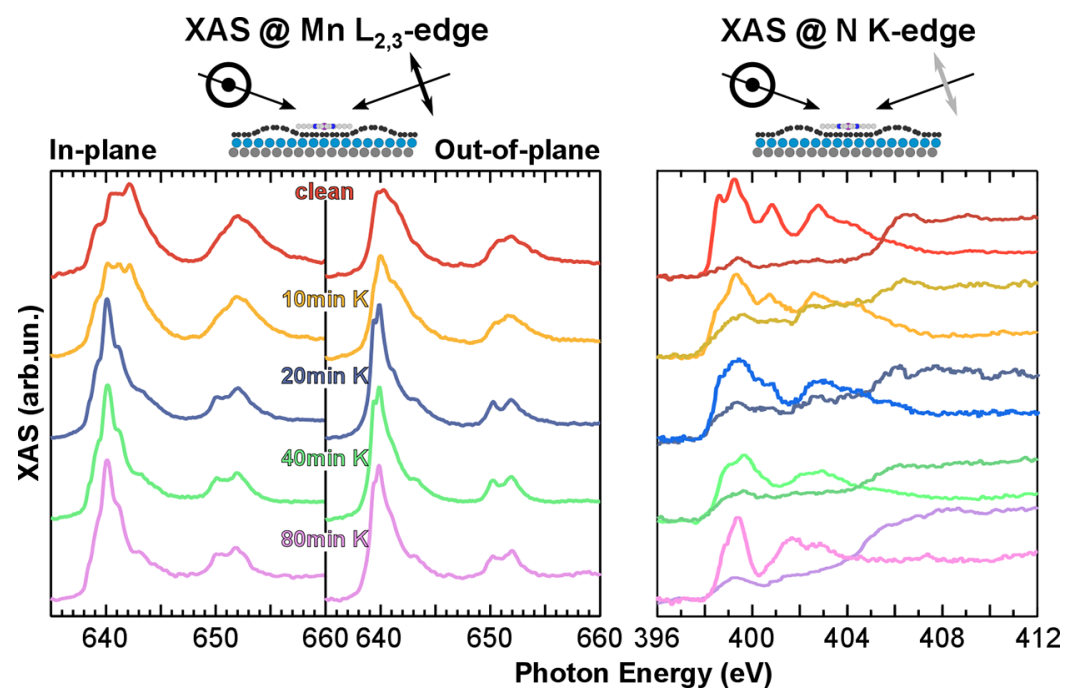

Figure 3. NEXAFS spectra at the $\mathrm{Mn} \mathrm{L}_{2,3}$ (left) and $\mathrm{N} \mathrm{K}$ (right) absorption edges, acquired with linearly polarized light for $\mathrm{MnPc} / \mathrm{Gr} / 1 \mathrm{ML} \mathrm{Co} /$ $\operatorname{Ir}(111)$ at increasing $\mathrm{K}$ doping. The measurements are performed with the sample kept at $100 \mathrm{~K}$.

line shape and an overall decrease of the total spectral intensity of the $\pi^{*}$ resonances suggest an electron doping at the macrocycle $\mathrm{N}$ sites. ${ }^{32,41}$ There is consensus in claiming that two stable configurations are found for K-doped TM-Pcs ( $\mathrm{TM}=\mathrm{Fe}, \mathrm{Ni}, \mathrm{Cu}, \mathrm{Zn}$ ), namely, $\mathrm{K}_{2} \mathrm{TM}-\mathrm{Pc}$ and $\mathrm{K}_{4} \mathrm{TM}-\mathrm{Pc}$, with electrons being donated mainly to molecular orbitals delocalized over the organic macrocycle. ${ }^{30,31}$ This evidence suggests that the electron doping induces a filling of the empty ligand states, affecting the crystal field exerted on the central $\mathrm{Mn}$ ion and determining a transition to a new Mn ground state configuration.

A one-to-one attribution to the new configuration of $\mathrm{Mn}$ orbitals upon $\mathrm{e}^{-}$doping gets complicated by the concomitant correlation effects at the open $3 \mathrm{~d}$ shell of the $\mathrm{Mn}$ core. To unveil the origin of the spin configuration before and after the alkali doping, multiplet calculations (see Experimental Methods and the Supporting Information for details) have been performed to simulate the $\mathrm{Mn} \mathrm{L}_{2,3}$ absorption edges and XMCD. A comparison between measured (left) and calculated (right) NEXAFS $\mathrm{L}_{2,3}$ spectra for both clean (top) and $\mathrm{K}$ fully doped (bottom) MnPc on $\mathrm{Gr} / \mathrm{Co} / \mathrm{Ir}(111)$ is shown in Figure 4. In order to simulate the ground-state configuration of the pristine $\mathrm{MnPc}$ molecule, a $\mathrm{D}_{4 \mathrm{~h}}$ crystal field symmetry has been assumed. The charge-transfer parameters are considered negligible, thanks to the decoupling effect of the Gr layer on top of the Co film. ${ }^{35}$ A good agreement between the experimental and calculated linearly polarized in-plane and out-of-plane XAS has been obtained with the crystal field parameters $10 D q=4.30, D t=0.79$, and $D s=0.49$, giving a ground state $B_{1 g}$ symmetry with $S=3 / 2$ and a $\left(e_{g}\right)^{2}\left(b_{2 g}\right)^{1}\left(a_{1 g}\right)^{2}\left(b_{1 g}\right)^{0}$ configuration, in agreement with the output of other multiplet calculations reported in the literature for analogous systems. ${ }^{42,43}$ For the doped system, an agreement between experimental and calculated XAS spectra has been obtained, imposing an overall marked reduction of the crystal field potential $(10 D q=1.08, D t=-0.10, D s=$ $0.15)$, resulting in an estimated $\left(\mathrm{e}_{\mathrm{g}}\right)^{2}\left(\mathrm{~b}_{2 \mathrm{~g}}\right)^{1}\left(\mathrm{a}_{1 \mathrm{~g}}\right)^{1}\left(\mathrm{~b}_{1 \mathrm{~g}}\right)^{1}$ orbital occupation sequence, with a high-spin $S=5 / 2$ configuration and an unvaried $\mathrm{Mn} 3 \mathrm{~d}^{5}$ occupation number. The magnetic dichroism is fairly well reproduced for the K-saturated $\mathrm{MnPc}$ (Figure 4) with the set of crystal field parameters previously reported, thus corroborating a $3 / 2 \rightarrow 5 / 2$ spin transition. The reduction of the crystal field parameters in the doped phase reveals a transition to an almost-octahedral symmetry environment of the $\mathrm{Mn}$ ion, suggesting a weaker $\mathrm{Mn}$-ligand interaction in the $n$-doped molecule with respect to the pristine one, in agreement with previous results reported for Li-doped MnPc molecules directly adsorbed on the $\operatorname{Ag}(100)$ surface. $^{21}$ As revealed by the $\mathrm{N}$ K-edge evolution upon $\mathrm{K}$ doping, the marked reduction of the crystal field potential can be ascribed to the result of charge donated to the organic ligands surrounding the $\mathrm{Mn}$ ions. The reduction of the crystal field potential is also an indication of an overall reduction of the orbital overlap between the $\mathrm{Mn}$ ion and the organic ligands, which in turn results in a hindered ligand-mediated super-exchange interaction channel between the Co and the Mn-ion magnetic moments. Furthermore, the higher spin ground state determines an increase of the magnetic moment, pinpointed by the higher XMCD signal and the fielddependent magnetization observed as a function of the $\mathrm{K}$ dosing.

The electronic modification of the organic ligands, as a consequence of $\mathrm{e}^{-}$doping, causes a strong reduction of the interaction between the organic cage and the central metal atom. The lowered ligand-metal interaction determines a weakening of the $\mathrm{Co}-\mathrm{Gr}-\mathrm{N}-\mathrm{Mn}$ super-exchange interaction path between the $\mathrm{Mn}$ ion and the Co layer, in consequence of the reduced electron-hopping probability in the hybridized orbitals that governs the super-exchange mechanism. ${ }^{34}$ This process in turn leads to a switch between a strong AFM to a weak FM coupling, eventually allowing the Mn ion magnetization to orient with the external field, independently of the Co layer one.

\section{CONCLUSIONS}

We demonstrate that the super-exchange mechanism that determines the magnetic state of a graphene-supported molecular spin interface can be tuned by electron doping of the molecular orbitals. The spin interface, constituted by $\mathrm{MnPc}$ molecules arranged on the rippled graphene membrane and slightly interacting with a Co intercalated layer, reveals a switching of the magnetic state from strong AFM to weak-field controllable FM, upon potassium doping. The $\mathrm{MnPc}$ 


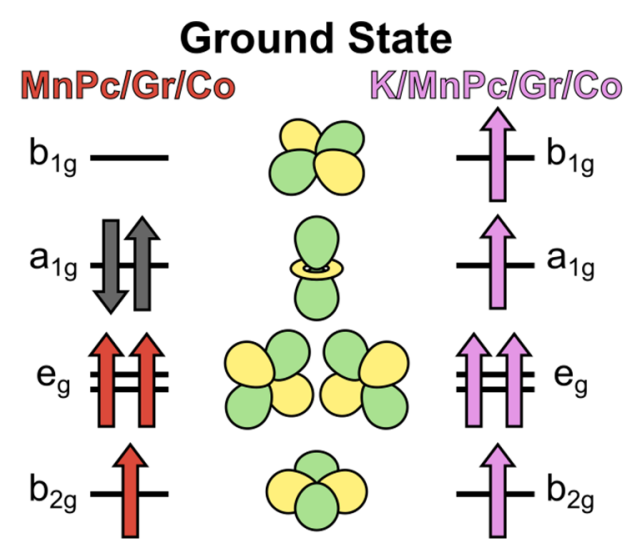

XAS @ Mn L 2,3 -edge
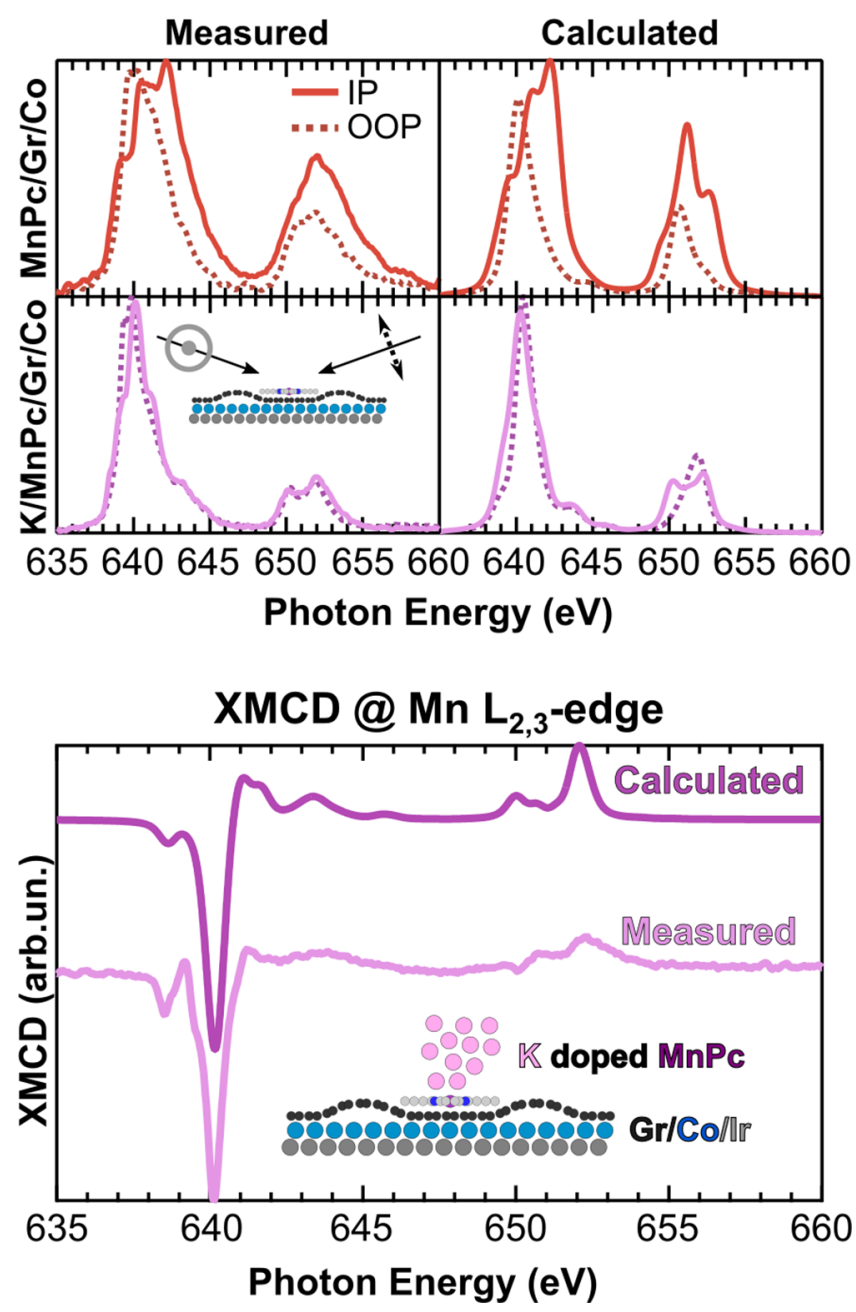

Figure 4. Top panel: Ground state configurations, as obtained by the theoretical simulation, for the clean (left) and K-doped (right) samples. Central panel: Comparison between the measured (left) and calculated (right) XAS spectra with in-plane (continuous lines) and out-of-plane (dashed lines) polarized light. Bottom panel: Experimental XMCD spectra for $\mathrm{K} / \mathrm{MnPc} / \mathrm{Gr} / \mathrm{Co}$, acquired at $\mathrm{NI}$ and with a $6 \mathrm{~T}$ magnetic field at the $\mathrm{Mn} \mathrm{L}_{2,3}$ absorption edge (top curve), compared with the resulting multiplet calculation (bottom curve).

molecular magnetic response is enhanced, changing the magnetic configuration from an intermediate spin $S=3 / 2$ to a high spin $S=5 / 2$ state. The resulting molecular spin interface presents a high magnetic polarizability mostly independent from the hard-magnet substrate, enabling possible applications as molecular spin-sensing devices. This model system demonstrates that metal phthalocyanines can be key enablers to explore tunable magnetic interactions, exploiting different super-exchange pathways, and that a new route can be opened to design and control the magnetic state of spin interfaces with an unprecedented role in the future of molecular spintronics.

\section{EXPERIMENTAL METHODS}

Sample Preparation. The $\operatorname{Ir}(111)$ surface was prepared by cycles of $\mathrm{Ar}^{+}$sputtering $(1.5 \mathrm{keV})$ and annealing $(1300 \mathrm{~K})$. The $\mathrm{Gr}$ layer was grown via chemical vapor deposition, i.e., by exposing the hot $\operatorname{Ir}(111)$ surface, kept at $1500 \mathrm{~K}$, to a $\mathrm{C}_{2} \mathrm{H}_{4}$ partial pressure of $2.0 \times 10^{-6}$ mbar. $^{44}$ Co intercalation was achieved by depositing metallic Co on the $\mathrm{Gr} / \operatorname{Ir}(111)$ surface at room temperature and subsequently annealing the $\mathrm{Co} / \mathrm{Gr} /$ $\operatorname{Ir}(111)$ sample up to $600 \mathrm{~K}^{45}$ The surface symmetry was checked with low-energy electron diffraction measurements, confirming the presence of a definite single-domain moiré pattern. MnPc molecules, sublimated from a commercially available powder (Sigma-Aldrich) previously purified by several hours of annealing in vacuum, have been deposited on top of the graphene membrane. The Co $\left(\rho=8.9 \mathrm{~g} / \mathrm{cm}^{3}\right)$ and $\mathrm{MnPc}\left(\rho=1.5 \mathrm{~g} / \mathrm{cm}^{3}\right)$ coverage were measured with a quartz crystal microbalance. $\mathrm{K}$ deposition was performed in the measurement chamber, with two SAES getters pockets in series kept at $5.5 \mathrm{~A}$, at a distance of $30 \mathrm{~cm}$ from the sample kept at $100 \mathrm{~K}$ during deposition. The pressure in the chamber during evaporation was in the low $10^{-10}$ mbar range.

X-ray Absorption Measurements. XMCD experiments were carried out at the BOREAS beamline of the ALBA Synchrotron Radiation Facility (Barcelona, Spain). The absorption signal was recorded in total electron yield mode and normalized to the photon flux as measured by a refreshed gold grid. ${ }^{46}$ The measurements were performed by impinging the sample with circularly polarized radiation at $0^{\circ}$ with respect to the surface normal. The sample was kept at low temperature (2.7 $\mathrm{K}$ as measured at the coldfinger of the cryostat) during the XMCD measurements. In the same experimental conditions, hysteresis loops were acquired by monitoring the XMCD signal at increasing (decreasing) magnetic field, normalized with respect to the pre-edge to avoid field-induced artifacts. Near-edge X-ray absorption fine structure (NEXAFS) spectra were collected by impinging the sample at grazing incidence $(\theta$ $=70^{\circ}$ ) with linearly polarized light, either parallel or perpendicular to the propagation direction.

Multiplet Calculations. Multiplet calculations were performed using the CTM4XAS package ${ }^{47}$ in a $\mathrm{C} 4 \mathrm{v}$ ligand field symmetry, in order to take into account the lowered symmetry of the surface-supported $\mathrm{MnPc}$ molecules. The crystal field parameters are reported in the Supporting Information and are summarized in the text.

\section{ASSOCIATED CONTENT}

\section{Supporting Information}

The Supporting Information is available free of charge at https://pubs.acs.org/doi/10.1021/acs.nanolett.0c04256.

Detailed materials related to the multiplet calculations to simulate the XAS measurement at the $\mathrm{Mn} L_{2,3}$ edges, including all the parameters (PDF) 
AUTHOR INFORMATION

\section{Corresponding Author}

Maria Grazia Betti - Physics Department, Sapienza University of Rome, 500185 Rome, Italy; $\odot$ orcid.org/0000-00026244-0306; Email: maria.grazia.betti@romal.infn.it

\section{Authors}

Giulia Avvisati - Physics Department, Sapienza University of Rome, 500185 Rome, Italy; ๑ orcid.org/0000-0002-3126838X

Pierluigi Gargiani - ALBA Synchrotron Light Source, 2-26 08290 Barcelona, Spain; ๑ orcid.org/0000-0002-66490538

Carlo Mariani - Physics Department, Sapienza University of Rome, 500185 Rome, Italy; @ orcid.org/0000-0002-79791700

Complete contact information is available at:

https://pubs.acs.org/10.1021/acs.nanolett.0c04256

\section{Notes}

The authors declare no competing financial interest.

\section{ACKNOWLEDGMENTS}

These experiments were performed at the BOREAS beamline at ALBA Synchrotron with the collaboration of ALBA staff and CALIPSOplus (grant 730872) funding. This work was partially supported by PRIN FERMAT (2017KFY7XF) from the Italian Ministery MIUR and by Sapienza Ateneo funds.

\section{REFERENCES}

(1) Cornia, A.; Seneor, P. The molecular way. Nat. Mater. 2017, 16, 505-506.

(2) Bogani, L.; Wernsdorfer, W. Molecular spintronics using singlemolecule magnets. Nat. Mater. 2008, 7, 179-186.

(3) Xiong, Z. H.; Wu, D.; Valy Vardeny, Z.; Shi, J. Giant magnetoresistance in organic spin-valves. Nature 2004, 427, 821-824.

(4) Dediu, V. A.; Hueso, L. E.; Bergenti, I.; Taliani, C. Spin routes in organic semiconductors. Nat. Mater. 2009, 8, 707-16.

(5) Gobbi, M.; Golmar, F.; Llopis, R.; Casanova, F.; Hueso, L. E. Room-Temperature Spin Transport in C60-Based Spin Valves. Adv. Mater. 2011, 23, 1609-1613.

(6) Santos, T.; Lee, J.; Migdal, P.; Lekshmi, I.; Satpati, B.; Moodera, J. Room-Temperature Tunnel Magnetoresistance and Spin-Polarized Tunneling through an Organic Semiconductor Barrier. Phys. Rev. Lett. 2007, 98, 3-6.

(7) Rakhmilevitch, D.; Sarkar, S.; Bitton, O.; Kronik, L.; Tal, O. Enhanced Magnetoresistance in Molecular Junctions by Geometrical Optimization of Spin-Selective Orbital Hybridization. Nano Lett. 2016, 16, 1741-1745.

(8) Sanvito, S. The rise of spinterface science. Nat. Phys. 2010, 6, $562-564$.

(9) Barraud, C.; Seneor, P.; Mattana, R.; Fusil, S.; Bouzehouane, K.; Deranlot, C.; Graziosi, P.; Hueso, L.; Bergenti, I.; Dediu, V.; Petroff, F.; Fert, A. Unravelling the role of the interface for spin injection into organic semiconductors. Nat. Phys. 2010, 6, 615-620.

(10) Kawahara, S. L.; Lagoute, J.; Repain, V.; Chacon, C.; Girard, Y.; Rousset, S.; Smogunov, A.; Barreteau, C. Large Magnetoresistance through a Single Molecule due to a Spin-Split Hybridized Orbital. Nano Lett. 2012, 12, 4558-4563.

(11) Raman, K. V.; Kamerbeek, A. M.; Mukherjee, A.; Atodiresei, N.; Sen, T. K.; Lazić, P.; Caciuc, V.; Michel, R.; Stalke, D.; Mandal, S. K.; Blügel, S.; Münzenberg, M.; Moodera, J. S. Interface-engineered templates for molecular spin memory devices. Nature 2013, 493, 509-513.

(12) Moorsom, T.; et al. Reversible spin storage in metal oxidefullerene heterojunctions. Science Advances 2020, 6, No. eaax1085.
(13) Campbell, V. E.; et al. Engineering the magnetic coupling and anisotropy at the molecule-magnetic surface interface in molecular spintronic devices. Nat. Commun. 2016, 7, 13646.

(14) Wende, H.; et al. Substrate-induced magnetic ordering and switching of iron porphyrin molecules. Nat. Mater. 2007, 6, 516-520.

(15) Wäckerlin, C.; Chylarecka, D.; Kleibert, A.; Müller, K.; Iacovita, C.; Nolting, F.; Jung, T. A.; Ballav, N. Controlling spins in adsorbed molecules by a chemical switch. Nat. Commun. 2010, 1, 61.

(16) Lodi Rizzini, A.; Krull, C.; Balashov, T.; Kavich, J. J.; Mugarza, A.; Miedema, P. S.; Thakur, P. K.; Sessi, V.; Klyatskaya, S.; Ruben, M.; Stepanow, S.; Gambardella, P. Coupling Single Molecule Magnets to Ferromagnetic Substrates. Phys. Rev. Lett. 2011, 107, 177205.

(17) Ormaza, M.; Abufager, P.; Verlhac, B.; Bachellier, N.; Bocquet, M.-L.; Lorente, N.; Limot, L. Controlled spin switching in a metallocene molecular junction. Nat. Commun. 2017, 8, 1974.

(18) Bartolomé, J.; Monton, C.; Schuller, I. K. In Molecular Magnets: Physics and Applications; Bartolomé, J., Luis, F., Fernández, J. F., Eds.; Springer: Berlin, 2014; pp 221-245.

(19) Gargiani, P.; Rossi, G.; Biagi, R.; Corradini, V.; Pedio, M.; Fortuna, S.; Calzolari, A.; Fabris, S.; Cezar, J. C.; Brookes, N. B.; Betti, M. G. Spin and orbital configuration of metal phthalocyanine chains assembled on the $\mathrm{Au}(110)$ surface. Phys. Rev. B: Condens. Matter Mater. Phys. 2013, 87, 165407.

(20) Mabrouk, M.; Savoyant, A.; Giovanelli, L.; Clair, S.; Hayn, R.; Ben Chaabane, R. Ligand Influence on Local Magnetic Moments in Fe-Based Metal-Organic Networks. J. Phys. Chem. C 2017, 121, 4253-4260.

(21) Stepanow, S.; Lodi Rizzini, A.; Krull, C.; Kavich, J.; Cezar, J. C.; Yakhou-Harris, F.; Sheverdyaeva, P. M.; Moras, P.; Carbone, C.; Ceballos, G.; Mugarza, A.; Gambardella, P. Spin Tuning of ElectronDoped Metal-Phthalocyanine Layers. J. Am. Chem. Soc. 2014, 136, 5451-5459.

(22) Krull, C.; Robles, R.; Mugarza, A.; Gambardella, P. Site- and orbital-dependent charge donation and spin manipulation in electrondoped metal phthalocyanines. Nat. Mater. 2013, 12, 337-343.

(23) Miedema, P. S.; van Schooneveld, M. M.; Bogerd, R.; Rocha, T. C. R.; Hävecker, M.; Knop-Gericke, A.; de Groot, F. M. F. Oxygen Binding to Cobalt and Iron Phthalocyanines As Determined from in Situ X-ray Absorption Spectroscopy. J. Phys. Chem. C 2011, 115, 25422-25428.

(24) Bartolomé, J.; Bartolomé, F.; Brookes, N. B.; Sedona, F.; Basagni, A.; Forrer, D.; Sambi, M. Reversible Fe Magnetic Moment Switching in Catalytic Oxygen Reduction Reaction of Fe-Phthalocyanine Adsorbed on $\operatorname{Ag}(110)$. J. Phys. Chem. C 2015, 119, 1248812495 .

(25) Stróżecka, A.; Soriano, M.; Pascual, J. I.; Palacios, J. J. Reversible Change of the Spin State in a Manganese Phthalocyanine by Coordination of CO Molecule. Phys. Rev. Lett. 2012, 109, 147202.

(26) Friedrich, R.; Hahn, T.; Kortus, J.; Fronk, M.; Haidu, F.; Salvan, G.; Zahn, D. R. T.; Schlesinger, M.; Mehring, M.; Roth, F.; Mahns, B.; Knupfer, M. Electronic states and the influence of oxygen addition on the optical absorption behaviour of manganese phthalocyanine. J. Chem. Phys. 2012, 136, 064704.

(27) Clack, D.; Hush, N.; Yandle, J. The ESR spectra of metal phthalocyanine negative ions. Chem. Phys. Lett. 1967, 1, 157-159.

(28) Clack, D. W.; Yandle, J. R. Electronic spectra of the negative ions of some metal phthalocyanines. Inorg. Chem. 1972, 11, 17381742.

(29) Clack, D.; Hush, N.; Woolsey, I. Reduction potentials of some metal phthalocyanines. Inorg. Chim. Acta 1976, 19, 129-132.

(30) Gargiani, P.; Calabrese, A.; Mariani, C.; Betti, M. G. Control of Electron Injection Barrier by Electron Doping of Metal Phthalocyanines. J. Phys. Chem. C 2010, 114, 12258-12264.

(31) Flatz, K.; Grobosch, M.; Knupfer, M. The electronic properties of potassium doped copper-phthalocyanine studied by electron energy-loss spectroscopy. J. Chem. Phys. 2007, 126, 214702.

(32) Calabrese, A.; Floreano, L.; Verdini, A.; Mariani, C.; Betti, M. $\mathrm{G}$. Filling empty states in a $\mathrm{CuPc}$ single layer on the $\mathrm{Au}(110)$ surface 
via electron injection. Phys. Rev. B: Condens. Matter Mater. Phys. 2009, $79,115446$.

(33) Avvisati, G.; Cardoso, C.; Varsano, D.; Ferretti, A.; Gargiani, P.; Betti, M. G. Ferromagnetic and Antiferromagnetic Coupling of Spin Molecular Interfaces with High Thermal Stability. Nano Lett. 2018, $18,2268-2273$.

(34) Avvisati, G.; Gargiani, P.; Mondelli, P.; Presel, F.; Baraldi, A.; Betti, M. G. Superexchange pathways stabilize the magnetic coupling of $\mathrm{MnPc}$ with Co in a spin interface mediated by graphene. Phys. Rev. B: Condens. Matter Mater. Phys. 2018, 98, 115412.

(35) Avvisati, G.; Gargiani, P.; Mondelli, P.; Presel, F.; Bignardi, L.; Baraldi, A.; Betti, M. G. Metal phthalocyanines interaction with Co mediated by a moiré graphene superlattice. J. Chem. Phys. 2019, 150, 054704.

(36) Jo, J.; Byun, J.; Lee, J.; Choe, D.; Oh, I.; Park, J.; Jin, M.; Lee, J.; Yoo, J. Emergence of Multispinterface and Antiferromagnetic Molecular Exchange Bias via Molecular Stacking on a Ferromagnetic Film. Adv. Funct. Mater. 2020, 30, 1908499.

(37) Gargiani, P.; Lisi, S.; Avvisati, G.; Mondelli, P.; Fatale, S.; Betti, M. G. Mixing of $\mathrm{MnPc}$ electronic states at the $\mathrm{MnPc} / \mathrm{Au}(110)$ interface. J. Chem. Phys. 2017, 147, 134702.

(38) Kroll, T.; Kraus, R.; Schönfelder, R.; Aristov, V. Y.; Molodtsova, O. V.; Hoffmann, P.; Knupfer, M. Transition metal phthalocyanines: Insight into the electronic structure from soft x-ray spectroscopy. J. Chem. Phys. 2012, 137, 054306.

(39) van der Laan, G.; Figueroa, A. I. X-ray magnetic circular dichroism-A versatile tool to study magnetism. Coord. Chem. Rev. 2014, 277-278, 95-129.

(40) Betti, M. G.; Gargiani, P.; Frisenda, R.; Biagi, R.; Cossaro, A.; Verdini, A.; Floreano, L.; Mariani, C. Localized and Dispersive Electronic States at Ordered $\mathrm{FePc}$ and $\mathrm{CoPc}$ Chains on $\mathrm{Au}(110)$. J. Phys. Chem. C 2010, 114, 21638-21644.

(41) Aristov, V.; Molodtsova, O.; Maslyuk, V.; Vyalikh, D.; Bredow, T.; Mertig, I.; Preobrajenski, A.; Knupfer, M. Electronic properties of potassium-doped FePc. Org. Electron. 2010, 11, 1461-1468.

(42) Stepanow, S.; Miedema, P. S.; Mugarza, A.; Ceballos, G.; Moras, P.; Cezar, J. C.; Carbone, C.; de Groot, F. M. F.; Gambardella, P. Mixed-valence behavior and strong correlation effects of metal phthalocyanines adsorbed on metals. Phys. Rev. B: Condens. Matter Mater. Phys. 2011, 83, 220401.

(43) Kitaoka, Y.; Sakai, T.; Nakamura, K.; Akiyama, T.; Ito, T. Magnetism and multiplets in metal-phthalocyanine molecules. J. Appl. Phys. 2013, 113, 17E130.

(44) Coraux, J.; N’Diaye, A. T.; Busse, C.; Michely, T. Structural Coherency of Graphene on $\operatorname{Ir}(111)$. Nano Lett. 2008, 8, 565-570.

(45) Pacilé, D.; Lisi, S.; Di Bernardo, I.; Papagno, M.; Ferrari, L.; Pisarra, M.; Caputo, M.; Mahatha, S. K.; Sheverdyaeva, P. M.; Moras, P.; Lacovig, P.; Lizzit, S.; Baraldi, A.; Betti, M. G.; Carbone, C. Electronic structure of graphene/Co interfaces. Phys. Rev. B: Condens. Matter Mater. Phys. 2014, 90, 195446.

(46) Barla, A.; Nicolás, J.; Cocco, D.; Valvidares, S. M.; HerreroMartín, J.; Gargiani, P.; Moldes, J.; Ruget, C.; Pellegrin, E.; Ferrer, S. Design and performance of BOREAS, the beamline for resonant X-ray absorption and scattering experiments at the ALBA synchrotron light source. J. Synchrotron Radiat. 2016, 23, 1507-1517.

(47) Stavitski, E.; de Groot, F. M. The CTM4XAS program for EELS and XAS spectral shape analysis of transition metal L edges. Micron 2010, 41, 687-694. 